\title{
Note on Self-Duality and the Kodama State
}

\author{
Alejandro Corich and Jerónimo Corted \\ Instituto de Ciencias Nucleares \\ Universidad Nacional Autónoma de México \\ A. Postal 70-543, México D.F. 04510, MEXICO
}

\begin{abstract}
An interesting interplay between self-duality, the Kodama (Chern-Simons) state and knot invariants is shown to emerge in the quantum theory of an Abelian gauge theory. More precisely, when a self-dual representation of the CCR is chosen, the corresponding vacuum in the Schrödinger representation is precisely given by the Kodama state. Several consequences of this construction are explored.

PACS numbers: 03.70.+k, 04.62.+v
\end{abstract}

\section{INTRODUCTION}

In a quantum gauge theory in $3+1$ dimensions, there is a natural background independent state that can be defined in the Schrödinger picture, namely, the exponential of the Chern-Simons form,

$$
\Psi_{\mathrm{K}}[A]=\exp \left(S_{\mathrm{cs}}\right)
$$

where $S_{\mathrm{cs}}=k \int_{\Sigma}\left(\frac{1}{2} A \wedge \mathrm{d} A+\frac{1}{3} A \wedge A \wedge A\right)$. This state is known in the (loop) quantum gravity community as the Kodama state, since it was first defined by Kodama in the context of mini-superspace cosmological models [1] (although it was first introduced by Jackiw in the context of Yang-Mills theory [2]). This state has been extensively studied by Smolin, who has argued that, in the case of $A$ a self-dual connection, the Kodama state represents a vacuum state centered around classical de Sitter space [3]. In a recent paper Witten has listed some of the features that make this state unsuitable as a realistic vacuum state in pure Yang-Mills theory [4]. In particular it is known that negative helicity states have negative energies (and norms). For an Abelian gauge theory, Witten has also constructed these states. More recently, Smolin and Freidel have argued that in gravity, the lessons from Yang-Mills theory do not necessarily apply: one has to consider the so called "reality conditions" [5]. In gravity with self-dual variables, this means choosing a physical inner product that renders the real observables Hermitian, and might solve the problem of non-normalizability of the state [5].

On the other hand, there has been some interest in the quantum theory of the Maxwell field, for self-dual connections, both in a holomorphic representation [6] and in the corresponding loop representation [7. In this later case it was found that the inner product for loop excitations was given by the Gauss linking number (of suitably smeared loops). The Linking number was also found to

\footnotetext{
*Electronic address: corichi@nuclecu.unam.mx
}

†Electronic address: cortez@nuclecu.unam.mx be prominent in the basic commutation relations (CCR) for (real) electric and magnetic fluxes [8].

The purpose of this note is twofold. On the one hand, we would like to make precise the construction outlined in 4] for Abelian fields, and to point to the interesting role that self-duality plays in the process. To be precise, we shall construct the Schrödinger representation for the CCR corresponding to a self-dual decomposition of the classical phase space, and find that the canonical vacuum state is given by the Kodama wave function. The decomposition leading to this state is metric-background independent and in a sense explains why the resulting quantum theory, with the Chern-Simons form as the vacuum state, is topological. This leads us to the second goal of this note, namely to make contact with the results found previously for self-dual fields [6, 7], and for the commutation relations [8], where simple link invariants were found to play key roles in the quantum theory. The interplay between self-duality, Chern-Simons and diffeomorphism invariance exhibited in this note allows us to put those previous results in a coherent picture.

The structure of this paper is as follows. In Sec 1 we consider the self-dual decomposition of the phase space that leads to the corresponding (Fock) quantization. In Sec. III we construct the corresponding Schrödinger representation, finding that the vacuum state is the Kodama wave function. We conclude this note in Sec. IV with a discussion.

Throughout this paper, we shall consider $c=1$, but will keep $e$ and $\hbar$ dimension-full.

\section{SELF-DUAL QUANTIZATION}

Let us consider a $U(1)$ gauge theory, that can be, for simplicity, thought to be the Maxwell theory. In the canonical approach the phase space $\Gamma$ is described by the magnetic potential $A_{a}$ and the electric field $E^{a}$, each point of $\Gamma$ defined by a pair $(A, E)$. For convenience, we introduce the electric vector potential $V_{a}$ such that $E^{a}=\epsilon^{a b c} \nabla_{b} V_{c}$, and $B^{a}=\frac{1}{\sqrt{h}} \tilde{\eta}^{a b c} \underline{F_{b c}}$ is the magnetic field on a hypersurface $\Sigma$. Recall that the basic Poisson 
Brackets are given by

$$
\left\{A_{a}(x), E^{b}(y)\right\}=4 \pi \delta_{a}^{b} \delta^{3}(x, y)
$$

We are using Gauss system of units for which $A_{a}$ carries the dimensions of $\sqrt{M / L}$ and $E^{a}$ has dimensions of $\sqrt{M / L^{3}}$. It is sometimes convenient to introduce smeared observables for the basic variables,

$$
A[f]:=\int_{\Sigma} \mathrm{d}^{3} x \sqrt{h} A_{a} f^{a} \quad, \quad E[g]:=\int_{\Sigma} \mathrm{d}^{3} x \sqrt{h} E^{a} g_{a}
$$

For these basic observables, the corresponding Canonical Commutation Relations (CCR) read,

$$
[\hat{A}[f], \hat{E}[g]]=4 \pi i \hbar \int_{\Sigma} \mathrm{d}^{3} x \sqrt{h} f^{a} g_{a} \hat{\mathrm{I}}
$$

It is known that in field theory on any curved background (including Minkowski), there is an infinite freedom in the representation of the CCR, or as is normally known, the choice of vacuum state [9]. In Minkowski space-time, Poincaré invariance selects the standard vacuum, but in the absence of symmetries the choice is not so straightforward.

In this note we will consider a particular representation that is very natural from the geometric viewpoint, even when it is not physically acceptable. This is the representation induced by the Hodge-duality operator $\star$. Recall that the $\star$-operator acts on the Faraday tensor $F_{a b}$ as ${ }^{\star} F_{a b}=\frac{1}{2} \epsilon_{a b c d} F^{c d}$. The relevant feature of the duality operator is that when squared it gives minus the identity: ${ }^{\star}\left({ }^{\star} F_{a b}\right)=-F_{a b}$. Therefore, the Hodge- $\star$ induces a complex structure $J$ on the phase space [10]. In terms of the canonical data, the complex structure takes the form,

$$
J \cdot\left(\begin{array}{c}
A_{a} \\
E^{b}
\end{array}\right)=\left(\begin{array}{c}
-V_{a} \\
B^{b}
\end{array}\right)
$$

One can also write it as the (matrix) operator,

$$
J=\left(\begin{array}{cc}
0 & -(\nabla \times)^{-1} \\
\nabla \times & 0
\end{array}\right)
$$

It is important to note that this self-dual decomposition of the phase space is completely background independent (or as sometimes is depicted, diffeomorphism invariant). This means that, given the manifold $\Sigma$ and initial data $(A, E)$ on $\Sigma$, the complex structure $J$ provides a canonical decomposition, independently of the metric $g_{a b}$ on the space-time $M$ and of the particular embedding of $\Sigma$ into $M$. In the standard Fock representation, the one particle Hilbert space $\mathcal{H}$ is built by completing the phase space $(A, E)$ with respect to the Hermitian inner product given by,

$$
\langle\cdot, \cdot\rangle=\frac{1}{2 \hbar} \mu(\cdot, \cdot)-i \frac{1}{2 \hbar} \Omega(\cdot, \cdot) .
$$

where the real inner product $\mu(\cdot, \cdot)=\Omega(J \cdot, \cdot)$ 9]. Here $\Omega(\cdot, \cdot)$ is the naturally defined symplectic two-form 10 , 11], that for canonical data $(A, E)$ reads,

$$
\Omega\left((A, E),\left(A^{\prime}, E^{\prime}\right)\right)=\int_{\Sigma} \sqrt{h} \mathrm{~d}^{3} x\left(E^{a} A_{a}^{\prime}-E^{\prime a} A_{a}\right) .
$$

\section{KODAMA STATE}

The corresponding Schrödinger representation can be easily obtained by a direct generalization of the result of Ref. [12] to the Maxwell field. Let us now summarize the main steps in the construction. The first step is to consider the generators of the Weyl algebra,

$$
\hat{W}[l]:=\exp \left[\frac{i}{\hbar} \hat{\Omega}(l, \cdot)\right]
$$

where

$$
\Omega(l, \cdot)=\Omega\left(\left(-g_{a}, f^{b}\right),\left(A_{a}, E^{b}\right)\right)=A[f]+E[g]
$$

is a general linear observable labelled by the pair $(-g, f)$.

The strategy in order to construct the Schrödinger representation is to employ a GNS construction for which an algebraic state $\omega$ is defined as,

$$
\omega(\hat{W}[l])=\exp \left[-\frac{\pi}{\hbar} \mu(l, l)\right]
$$

In the resulting quantum theory, the quantity $\omega(\hat{W}[l])$ corresponds to the vacuum expectation value of the Weyl generators $\langle\hat{W}[l]\rangle_{0}$. For details of the construction see [12], and for a summary and some discussion see 13]. In order to arrive at the quantum measure, one considers the Weyl generators corresponding to configuration observables, in such a way that we have,

$$
\exp \left[-\frac{\pi}{\hbar} \mu\left(\left(0, f^{a}\right),\left(0, f^{a}\right)\right)\right]=\int_{\overline{\mathcal{C}}} \mathrm{d} \mu \exp \left[-\frac{i}{\hbar} A[f]\right]
$$

What is to say, that the Fourier transform $\chi[f]$ of the measure $\mathrm{d} \mu$ given by,

$$
\chi_{\mu}[f]=\int_{\overline{\mathcal{C}}} \mathrm{d} \mu \exp \left[-\frac{i}{\hbar} A[f]\right] .
$$

takes the form

$$
\chi_{\mu}[f]=\exp \left[-\frac{\pi}{\hbar} \int_{\Sigma} \mathrm{d}^{3} x \sqrt{h} f^{a}\left(\epsilon^{a b c} \nabla_{b}\right)^{-1} f^{c}\right]
$$

Now, the general theory of measures 14] assures us that the corresponding measure takes the form,

$$
\text { "d } \mu=\exp \left[-\frac{1}{4 \pi \hbar} \int_{\Sigma} \mathrm{d}^{3} x \sqrt{h} \epsilon^{a b c} A_{a} \nabla_{b} A_{c}\right] \mathcal{D} A ",
$$

But since $\int_{\Sigma} \mathrm{d}^{3} x \sqrt{h} \epsilon^{a b c} A_{a} \nabla_{b} A_{c}=\frac{1}{2} \int_{\Sigma} A \wedge \mathrm{d} A$, we have that,

$$
" \mathrm{~d} \mu=\exp \left[-\frac{1}{8 \pi \hbar} \int_{\Sigma} A \wedge \mathrm{d} A\right] \mathcal{D} A "
$$

If we consider a homogeneous measure, then the vacuum "absorbs" the measure and becomes non-trivial [15]. Thus we arrive at the vacuum state given by,

$$
\Psi_{0}[A]=\exp \left[-\frac{1}{16 \pi \hbar} \int_{\Sigma} A \wedge \mathrm{d} A\right]
$$


which is the so-called Kodama state. Note that we have been working in the "kinematical phase space", namely before imposing Gauss' law. However, the resulting representation is gauge invariant and naturally projects down to the reduced phase space.

Let us now explore this representation a bit. The basic observables are represented as,

$$
\hat{A}[f] \cdot \Psi[A]=(A[f] \Psi)[A],
$$

and

$$
\hat{E}[g] \cdot \Psi=-i 4 \pi \hbar \int_{\Sigma} \mathrm{d}^{3} x g_{a}\left(\frac{\delta}{\delta A_{a}} \Psi\right)
$$

Now, the vacuum state satisfies the following self-duality property:

$$
\left(\hat{B}^{a}+i \hat{E}^{a}\right) \cdot \Psi_{0}[A]=\left[B^{a}+i\left(-4 i \pi \hbar \frac{\delta}{\delta A_{a}}\right)\right] \Psi_{0}[A]=0
$$

This is the expected behavior of the Kodama state. Furthermore, it is an eigenstate of the Hamiltonian with zero eigenvalue as expected [4]. It is important to note that this self-dual property of the quantum vacuum state is satisfied for the Kodama state for real $\mathrm{U}(1)$ connections (as opposed to self-dual connections [6]).

Let us now consider Wilson loops functions $h_{\alpha}[A]$, labelled by loops $\alpha$, of the form

$$
h_{\alpha}[A]=\exp \left(i \frac{e}{\hbar} \oint_{\alpha} A\right)
$$

An obvious question one can ask is for the vacuum expectation value of such operators,

$$
\left\langle\hat{h}_{\alpha}\right\rangle_{0}=\int_{\overline{\mathcal{C}}} \mathcal{D} A \exp \left(-\frac{1}{8 \pi \hbar} \int_{\Sigma} A \wedge \mathrm{d} A\right) \exp \left(i \frac{e}{\hbar} \oint_{\alpha} A\right)
$$

This question has been extensively discussed in the literature (for a pedagogical account see [16]), and it is agreed that, after a framing, the quantity coincides with the self-linking of $\alpha$. If we now consider the "two loop function", $\left\langle\hat{h}_{\alpha} \hat{h}_{\beta}\right\rangle_{0}$, one should expect that this quantity should also be a knot invariant, related to the Gauss Linking number of the loops $\alpha$ and $\beta$ [16]. We recover then the intriguing result that the inner product between loop states is a knot invariant, when using self-dual connections [7]. That this quantum theory incorporates the linking number can be seen from the fact that the inner product between two 1-loop states $\hat{A}[\alpha]|0\rangle:=\left(\widehat{\oint_{\alpha} A}\right)|0\rangle$ and $\hat{A}[\beta]|0\rangle:=\left(\widehat{\oint_{\beta} A}\right)|0\rangle$ is given by the $\mu$ inner product on $\mathcal{H}[15]$. This quantity then takes the form,

$$
\begin{aligned}
\langle\hat{A}[\alpha] \hat{A}[\beta]\rangle_{0} & =\frac{2 \pi}{\hbar} \mu(F[\alpha], F[\beta]) \\
& =\frac{2 \pi}{\hbar} \int_{\Sigma} \mathrm{d}^{3} x F^{a}[\alpha, x)\left(\epsilon^{a b c} \nabla_{b}\right)^{-1} F^{c}[\beta, x)
\end{aligned}
$$

1. In retrospect, one might argue that the fact that
one recovers the results of $[7]$ is not entirely un-
expected. After all, the Schrödinger representa-
tion with a vertical polarization for the Hodge-dual

1. In retrospect, one might argue that the fact that
one recovers the results of [7] is not entirely un-
expected. After all, the Schrödinger representa-
tion with a vertical polarization for the Hodge-dual

1. In retrospect, one might argue that the fact that
one recovers the results of [7] is not entirely un-
expected. After all, the Schrödinger representa-
tion with a vertical polarization for the Hodge-dual

1. In retrospect, one might argue that the fact that
one recovers the results of [7] is not entirely un-
expected. After all, the Schrödinger representa-
tion with a vertical polarization for the Hodge-dual

where $F^{a}[\alpha, x)=\oint_{\alpha} \mathrm{d} \alpha^{a} \delta^{3}(\alpha, x)$ is the form factor of the loop $\alpha$, such that $\int_{\Sigma} \mathrm{d}^{3} x A_{a}(x) F^{a}[\alpha, x)=\oint_{\alpha} A$. In Ref. [8] it was shown that the Right Hand Side of (3.14) is indeed given by the Gauss linking number of $\alpha$ and $\beta$. Thus, we get:

$$
\langle\hat{A}[\alpha] \hat{A}[\beta]\rangle_{0}=\frac{2 \pi}{\hbar} \mathcal{G} \mathcal{L}(\alpha, \beta) .
$$

One could also consider the so called r-holonomies namely suitably smeared holonomies-, as the relevant configuration observables [17]. One would then get, instead of (3.15), properly generalized linking numbers as in [7].

\section{DISCUSSION}

Let us summarize our construction. We started with a complex structure on phase space defined by the Hodgedual on the space-time and, by means of a GNS construction, ended with the vacuum state given by the Kodama State. From this perspective, it is clear why this state has several problems. To begin with, the duality complex structure $J$ is not compatible with the symplectic structure $\Omega$, which means that the corresponding one particle Hilbert space $\mathcal{H}$ in the Fock construction has negative norm states. Furthermore, the operators that define the Fourier transform of the measure do not satisfy the positivity requirements: the measure is non-Gaussian, and non-normalizable.

Perhaps the most important question is whether these results on Abelian gauge theories can teach us something about the non-Abelian case, and in particular about gravity. Clearly we can say nothing about the issue of how a physical inner product on the space of solutions to the constraints might affect the normalizability of the state. However, we can still learn something from this simple model. What we have seen is that we do not need to introduce self-dual variables and complicated reality conditions in order to get a "self dual" representation and vacuum state [6]; our variables were always real. The selfduality in the resulting quantum theory came from the quantum representation, in this case due to the choice of complex structure on phase space. One might hope that this new perspective on the problem may shed some light in the goal of constructing physically relevant vacuum states and representations for gravity, without the need to introduce self-dual complex connections. Note also that even when we have considered a pure Abelian gauge field, this construction can also be done for linearizations of Yang-Mills [5] and gravity theories [18].

Let us end with two remarks: 
complex structure should be equivalent to a holomorphic representation with the self-dual polarization as in [6] and 7].

2. Nevertheless, it is interesting to see the non-trivial interplay between self-duality and diffeomorphism invariance that this model possesses. It has been known for some time that the symplectic structure $\Omega($, ) that appears in the inner product (2.6) is diffeomorphism invariant, a fact that is reflected in the Commutation Relations of the theory for the right kind of (electric and magnetic flux) variables
8]. In this note we have seen that when the other part of Eq. (2.6), namely the real inner product $\mu(,$,$) , is also diffeomorphism invariant, then we re-$ cover a fully topological quantum theory, at least at the kinematical level.

\section{Acknowledgments}

This work was supported in part by DGAPA-UNAM grant No. IN112401 and by CONACyT grant J32754-E.
[1] H. Kodama, "Holomorphic Wave Function Of The Universe," Phys. Rev. D 42, 2548 (1990).

[2] R. Jackiw, "Topological Investigations in Quantized Gauge Theories" in Current Algebra and Anomalies, Ed. S.B. Treiman et al (World Scientific, 1985).

[3] For a recent review see: L. Smolin, "Quantum gravity with a positive cosmological constant," arXiv:hep-th/0209079

[4] E. Witten, "A note on the Chern-Simons and Kodama wavefunctions," arXiv:gr-qc/0306083

[5] L. Freidel and L. Smolin, "The linearization of the Kodama state," arXiv:hep-th/0310224

[6] A. Ashtekar, C. Rovelli and L. Smolin, "Selfduality And Quantization," J. Geom. Phys. 8, 7 (1992) arXiv:hep-th/9202079.

[7] A. Ashtekar and A. Corichi, "Photon inner product and the Gauss-linking number," Class. Quant. Grav. 14, A43 (1997) arXiv:gr-qc/9608017.

[8] A. Ashtekar and A. Corichi, "Gauss linking number and electro-magnetic uncertainty principle," Phys. Rev. D 56, 2073 (1997) arXiv:hep-th/9701136.

[9] R.M. Wald, Quantum Field Theory in Curved Spacetime and Black Hole Thermodynamics (Chicago University Press, Chicago, 1994).

[10] A. Corichi, Rev. Mex. Fis. 44, 402 (1998).
arXiv:physics/9804018.

[11] C. Crnkovic and E. Witten, in Three hundred years of gravitation, Cambridge U. Press (1987).

[12] A. Corichi, J. Cortez, H. Quevedo, "Schrödinger representation for a scalar field on curved spacetime," Phys. Rev. D 66, 085025 (2002) arXiv:gr-qc/0207088.

[13] A. Corichi, J. Cortez and H. Quevedo, "Note on canonical quantization and unitary equivalence in field theory," Class. Quant. Grav. 20, L83 (2003) arXiv:gr-qc/0212023.

[14] Y.Yamasaki, Measures on infinite dimensional spaces (World Scientific, 1985).

[15] A. Corichi, J. Cortez and H. Quevedo, "On the relation between Fock and Schrödinger representations for a scalar field," arXiv:hep-th/0202070

[16] L.H. Kauffman, Knots and Physics (World Scientific, 1991)

[17] M. Varadarajan, "Fock representations from U(1) holonomy algebras," Phys. Rev. D 61, 104001 (2000) arXiv:gr-qc/0001050.

[18] M. Varadarajan, "Gravitons from a loop representation of linearised gravity," Phys. Rev. D 66, 024017 (2002) arXiv:gr-qc/0204067. 